Relations industrielles

Industrial Relations

\title{
Gareth Morgan, Images de l'organisation
}

\section{Laurent Bélanger}

Volume 46, numéro 1, 1991

URI : https://id.erudit.org/iderudit/050663ar

DOI : https://doi.org/10.7202/050663ar

Aller au sommaire du numéro

Éditeur(s)

Département des relations industrielles de l'Université Laval

ISSN

0034-379X (imprimé)

1703-8138 (numérique)

Découvrir la revue

Citer ce compte rendu

Bélanger, L. (1991). Compte rendu de [Gareth Morgan, Images de

l'organisation]. Relations industrielles / Industrial Relations, 46(1), 242-244.

https://doi.org/10.7202/050663ar

Tous droits réservés (C) Département des relations industrielles de l'Université Laval, 1991
Ce document est protégé par la loi sur le droit d'auteur. L'utilisation des services d'Érudit (y compris la reproduction) est assujettie à sa politique d'utilisation que vous pouvez consulter en ligne.

https://apropos.erudit.org/fr/usagers/politique-dutilisation/ 
Loi annotée sur les décrets de convention collective, par Patrick de Niverville et Claude CARIGNAN, Montréal, Wilson et Lafleur ltée., 1990, 209 p., ISBN 2-89127-154-8

Bien que la Loi sur les décrets de convention collective ne soit pas une loi d'application générale de notre droit du travail, elle régit quand même les conditions de travail de 140000 salariés à l'emploi de 20000 employeurs.

Cette publication comprend le texte de la loi, une table de jurisprudence ainsi que les textes de cinq jugements inédits.

Sous chaque article de la loi, et même parfois sous chaque paragraphe d'un même article, les auteurs ont rapporté plusieurs décisions rendues par les tribunaux ainsi qu'un certain nombre de références à la doctrine. Les auteurs ont même pris soin, non seulement de regrouper ces nombreuses sources de renseignements en fonction des textes de loi auxquels elles se rapportent, mais ils les ont souvent subdivisées en divers thèmes à l'intérieur d'une même disposition. Avant chacun des groupes ou sous-groupes de jurisprudence ou de doctrine rapportées, les auteurs ont pris soin de faire un bref résumé du contenu de ces décisions. D'ailleurs, des passages d'un certain nombre de ces dernières sont rapportés lorsque cela est pertinent. Il ne s'agit donc pas uniquement d'une compilation de citations des décisions des tribunaux. Les textes qui font état du contenu de ces dernières sont d'une grande utilité pour la compréhension de la portée des dispositions de la loi.

Cette publication constitue donc une mine très importante de renseignements.

Il est bien certain qu'un ouvrage de ce genre constitue nécessairement une certaine simplification de la matière abordée, et en ce sens, il faut être conscient qu'il y a certains risques à ne consulter que des résumés qui ne peuvent rendre compte de toutes les nuances des décisions rapportées. Il faut cependant admettre que le but de l'ouvrage n'est pas de traiter un sujet de façon exhaustive avec toutes les nuances qui s'imposent. Considérée comme une loi annotée, il ne fait aucun doute que cette publication atteint de façon parfaite son but qui est celui de renseigner le lecteur efficacement et rapidement sur les principales questions qui se posent au sujet des dispositions de la loi et sur les sources jurisprudentielles susceptibles de préciser le texte de cette dernière.

La présentation matérielle est d'une grande qualité. La table de jurisprudence ainsi que les textes de cinq jugements inédits sont aussi autant d'éléments qui rendent cet ouvrage très utile pour les praticiens du droit, les administrateurs de comités paritaires, les conseillers syndicaux et les employeurs.

Jean-Louis DUBÉ

Université de Sherbrooke

Images de l'organisation, par Gareth MoRGAN, Les Presses de l'Université Laval et les Éditions Eska, Québec, 1989, 556 p., ISBN 2-7637-7184-X (PUL) et ISBN 2-86911-026-X (Eska).

On l'aura reconnu, il s'agit là d'une traduction de l'ouvrage intitulé Images of Organization du professeur Gareth Morgan, en voie de devenir le meilleur traité de la décennie sur la nature et le fonctionnement des organisations. Les nombreuses recensions faites à ce jour y voient une contribution majeure dans le monde anglophone depuis la parution de l'ouvrage de Herbert Simon: Les organisations, problèmes psycho-sociologiques, dont la publication originale en anglais date de 1958. Dans le monde francophone, il faut signaler au moins un ouvrage comparable, celui de Michel Crozier et Erhard Friedberg: L'acteur et le système: les contraintes de l'action collective. 
La traduction, publiée dans la collection «Sciences de l'administration» a été effectuée par Solange Chevrier-Vouvé et Michel Audet de la Faculté des sciences de l'administration de l'Université Laval. Soulignons ici le travail formidable fait par les deux traducteurs, manifestant un souci constant de trouver les termes exacts, évitant de tomber dans la facilité qui consisterait à placer entre guillemets les termes anglais plus connus et couramment utilisés dans la langue de Molière.

Ceux qui sont à la recherche d'alternatives à la vision macaniciste et bureaucratique des organisations seront bien servis par la lecture de cet ouvrage. En effet, tout en accordant une place non négligeable au modèle de l'organisation-machine aux engrenages bien huilés, l'auteur propose d'autres visions des organisations:

- L'organisation envisagée sous l'angle d'un organisme vivant ou d'un système ouvert, l'auteur utilise ici l'approche système et la théorie de la contingence pour faire ressortir à la fois la nécessité de gérer convenablement l'interface organisation-environnement tout en assurant à l'interne une certaine stabilité de fonctionnement.

- L'organisation vue sous l'angle d'un cerveau en mettant en évidence l'apport de la cybernétique, des théories de l'apprentissage et des principes de l'holographie.

- L'organisation envisagée sous l'angle d'une culture, c'est-à-dire d'un ensemble de croyances, de valeurs, de représentations plus ou moins partagées par les membres qui viennent façonner, voire même, effectuer une régulation des conduites.

- L'organisation vue sous l'angle d'un système de gouvernement ou d'activité politique. L'auteur reprend ici les concepts d'autocratie, de démocratie (directe ou de représentation) pour en faire une application à la lecture des réalités organisationnelles où la collaboration alterne avec la recherche du pouvoir et la poursuite d'intérêts conflictuels.

- L'organisation envisagée sous l'angle d'une prison psychique. Il s'agit ici, on peut facilement en convenir, d'une métaphore ou d'une vision peu connue dans l'étude des organisations qui renvoie aux théories freudiennes de l'inconscient. Soulignons, en passant, la description faite de l'enfance de Taylor et de son obsession pour le contrôle. F. Taylor, doit-on le rappeler, est le père de l'organisation scientifique du travail.

- L'organisation envisagée comme flux et transformation. On découvre ici à l'aide d'une reformulation de la théorie des systèmes que les organisations ont une capacité de se transformer de l'intérieur, sans aide, à un point tel que la frontière organisation-environnement s'estompe et qu'on aurait exagéré l'importance de ce dernier.

- L'organisation envisagée sous l'angle d'un instrument de domination. C'est le prolongement de la métaphore de l'organisation système de gouvernement sur son aspect, cependant, plutôt «répugnant» pour reprendre le terme utilisé par les traducteurs.

La présentation de chacune des images ou métaphores est suivie d'une courte analyse de leurs forces et faiblesses. De plus, un dernier chapitre comprend des notes bibliographiques, c'est-à-dire une présentation des contributions de d'autres auteurs qui ont servi à appuyer la description des images. Ce chapitre, dans sa version française, comprend une multitude d'ajouts aux contributions de caractère anglophone qui traitent des mêmes sujets. Chaque fois qu'un ouvrage de langue anglaise comporte une traduction, les traducteurs et leurs collaborateurs le signalent, de sorte que la bibliographie en français est beaucoup plus abondante que la bibliographie originale. De plus, cette bibliographie est suivie d'un lexique anglais-français des termes courants en sciences administratives. 
Dans l'ensemble, la contribution originale de l'auteur consiste à réunir dans un seul volume l'apport de disciplines, tant des sciences humaines que de la physique et de la biologie, à l'analyse et la compréhension des organisations. En effet, on ne saurait accéder à une connaissance convenable des structures et du fonctionnement des organisations en privilégiant une vision en particulier et en se privant ainsi de l'éclairage fourni par d'autres métaphores. Le recours à diverses images permet une analyse plus raffinée des problèmes auxquels font face les organisations et facilite également la recherche de solutions plus appropriées.

Laurent BÉLANGER

Université Laval

Psychologie et organisations - L'individu dans son milieu de travail, par Léandre MAILLET, publié dans la Collection «Psychologie Industrielle et Organisationnelle», sous la direction de Yvan Bordeleau, Montréal, Agence d'ARC, 1988, 576 p., ISBN 2-89022-124-5

Comme l'écrit fort justement l'auteur sur la jaquette de son ouvrage «ce livre tente d'apprivoiser le lecteur face aux principaux aspects humains de l'organisation» et, pourrions-nous ajouter, particulièrement pour ceux et celles qui abordent l'étude des organisations et des sciences sociales. C'est un ouvrage de facture classique. L'auteur puise aux différentes disciplines des sciences humaines, davantage à celle de la psychologie appliquée au monde des organisations. Il se situe à cet égard dans la foulée des quelque six ouvrages parus en français ces dernières années.

Léandre Maillet parvient à intégrer, dans une perspective systémique, les principales données de la psychologie moderne pour mieux en comprendre les comportements de l'individu dans son milieu de travail. Le support bibliographique est impressionnant: les références sont nombreuses, pertinentes et récentes. Il aurait été assurément intéressant pour le lecteur francophone d'en trouver davantage en français, mais c'est là un état de fait qui échappe en partie à l'auteur.

Après avoir présenté un aperçu historique de la notion de comportement organisationnel, l'auteur s'attarde par la suite aux facteurs individuels (la perception, la communication, la motivation, l'apprentissage) pour ensuite aborder les facteurs à caractère social liés à l'organisation et au fonctionnement des groupes dans l'organisation (la dynamique des groupes, les conflits inter groupes, le pouvoir et le leadership). Ce manuel traite dans ses derniers chapitres du changement et du développement dans les organisations.

Le lecteur qui y chercherait une vision nouvelle, originale ou renouvelée du fonctionnement de l'individu en milieu organisationnel s'en trouvera probablement déçu comme d'ailleurs celui qui y chercherait des données inédites de l'auteur. Ce n'est pas la fin poursuivie par Léandre Maillet. Il vise un objectif essentiellement pédadogique en support à l'enseignement de ces matières. Il consigne et intègre dans un manuel les principales données de la psychologie moderne appliquées au monde des organisations afin de mieux en comprendre certains principes régissant le comportement individuel dans le cadre de ses activités de travail. Pour parvenir à cette visée pédagogique à chaque chapitre il fixe les objectifs d'apprentissage poursuivis, fait une mise en situation pour introduire la matière, soumet au lecteur des sujets de discussion et des études de cas. C'est ce qui fondamentalement lui donne son caractère de manuel en support à l'enseignement. 